\title{
Response time and click position: cheap indicators of preferences
}

\author{
Fadong Chen ${ }^{1,2,3} \cdot$ Urs Fischbacher ${ }^{1,2}$
}

Received: 29 November 2015/Revised: 26 August 2016/Accepted: 29 August 2016/

Published online: 14 September 2016

(C) Economic Science Association 2016

\begin{abstract}
This paper investigates how process data like response time and click position relates to economic decisions. We use a social value orientation experiment, which can be considered as a prototypical multi-attribute decision problem. We find that in the social value orientation task more individualistic subjects have shorter response times than prosocial subjects. Individualistic subjects click more often on their own payoffs than on the others' payoffs, and they click more often on their own payoffs than prosocial subjects. Moreover, the response time information and the click position information are complementary in explaining subjects' preferences. These results show that response times and click positions can be used as indicators of people's preferences.
\end{abstract}

Keywords Click position $\cdot$ Response time $\cdot$ Social preferences $\cdot$ Experiment

JEL Classification C72 - C91 - D87

Electronic supplementary material The online version of this article (doi:10.1007/s40881-016-0026-6) contains supplementary material, which is available to authorized users.

Urs Fischbacher

Urs.Fischbacher@uni-konstanz.de

Fadong Chen

Fadong.Chen@uni-konstanz.de

1 Department of Economics, University of Konstanz, Universitätsstr. 10, 78457 Konstanz, Germany

2 Thurgau Institute of Economics, Hauptstr. 90, 8280 Kreuzlingen, Switzerland

3 Graduate School of Decision Science, University of Konstanz, Universitätsstr. 10, 78457 Konstanz, Germany 


\section{Introduction}

There is an increasing interest in using process data to get a better understanding of economic decision making. Different methods such as response times (Rubinstein 2007), pupil dilations (Wang et al. 2010), eye movements (Reutskaja et al. 2011) and even neural activity (Smith et al. 2014) have been introduced and have shown significant correspondences between this kind of data and people's decisions. These methods vary significantly in their complexity and some of them are quite costly. We show that simple by-product data in lab or online economic experiments, such as response times and click positions, can provide information about people's preferences. With a proper design, this data can be collected at almost no cost. While response time analysis has been used frequently (for a review, see Spiliopoulos and Ortmann 2015), click positions have received attention mainly in computer science. There, click positions are analyzed routinely, in particular for the optimization of web pages (Guo et al. 2009; Liu et al. 2010). However, these studies use the click position only to determine the choice. We study what attributes of a choice subjects click on and show that the personally attractive attributes of a choice have a higher probability to be clicked.

Specifically, we use a social value orientation (SVO) experiment in which the response times and the click positions are recorded. The SVO task consists of several decision situations, each consisting of a menu of allocations between the deciding subject and an anonymous partner. Thus, the SVO measures how much people care about their own as compared to the other player's welfare. The SVO task represents a prototypical situation in which people have to assess and trade off different attributes of an option-in the case of the SVO their own vs the other's payoff. Our results show that more prosocial subjects take longer to make their decisions in the SVO task. Concerning the click position, we find that individualistic subjects click more often on their own payoffs than on the others' payoffs, and they click more often on their own payoffs than prosocial subjects. Moreover, the response time information and the click position information are complementary in explaining subjects' SVO. Thus, response time and click position can be used as indicators of people's preferences.

Response time has increasingly been used in economic experiments (Hutcherson et al. 2015; Krajbich et al. 2010, 2014), in particular as an indicator of whether a decision is made intuitively or deliberatively (Achtziger and Alós-Ferrer 2014; Krajbich et al. 2015; Piovesan and Wengström 2009; Rand et al. 2012; Schulz et al. 2014). Most closely related to our study is Fiedler et al. (2013), who use eyetracking technology to investigate the underlying processes of social decision making. They find that differences in SVO are accompanied by consistent differences in information search and response times. In another study by Liebrand and McClintock (1988) they find that the processing times vary systematically between different types of subjects. Specifically, cooperators and competitors are slower than altruists and individualists. There is a tradition of using the mouse to track the information search process or information processing. The most prominent 
example is probably MouseLab (Brocas et al. 2014; Payne et al. 1993). ${ }^{1}$ In this environment, subjects access the information hidden behind boxes on the computer screen by moving the cursor over the boxes. Another method that uses the natural interaction is "response dynamics" (Kieslich and Hilbig 2014; Koop and Johnson 2011; Spivey et al. 2005). In this paradigm, the mouse response is tracked as subjects move from a central location to one of two disparately spaced options. The curvature of the mouse movement is taken as an indicator of cognitive conflicts and more curved response trajectories indicate stronger conflicts.

We confirm previous evidence that the response time correlates with subjects' preferences and find that the click position also correlates with subjects' preferences. The click position provides much less detailed information than MouseLab since it does not record the information search process, but it has the advantage that it records natural behavior while in MouseLab people need to move the mouse explicitly to acquire information. In this sense, the analysis of the click position is similar to the response dynamics paradigm, which also uses the computer interaction as an additional source of information. Our study highlights the potential benefits of recording response times and click positions in economic experiments involving multiple attribute decision making and using this data to infer people's preferences.

\section{Experimental design and procedure}

The experiment is built on the SVO Slider Measure by Murphy et al. (2011). In this task, subjects make a series of allocation decisions. In each allocation decision, the subject chooses one out of nine allocations involving varying payoffs for herself and another anonymous participant. A screenshot of one item is shown in Fig. 1. In this example, the subject can choose one of nine options from the most individualistic distribution on the right to the most altruistic distribution on the left. In the SVO task, there are six primary items that allow the assessment of the subject's concern for the payoff of the other player. The sum of the chosen allocations determines a vector in the plane of the own and the other player's payoff and provides a scale of SVO that indicates the concern for the welfare of others. ${ }^{2}$ In addition, there are nine secondary items, which makes it possible to distinguish the prosocial motives of inequality aversion and efficiency. ${ }^{3}$ We do not refer to this distinction in our study, but we use the secondary items to check the robustness of our results.

The subjects made their decisions by clicking on the allocation that they preferred most by clicking on one of the dark grey areas. For each decision, we recorded the subject's response time and the coordinate of the position that the subject clicked. This shows whether the subject clicked on the side of their own payoff or on the side of the payoff of the other player. Before the distributional decision, the subjects needed to click on a button that appeared in the lower region

\footnotetext{
1 For details about MouseLab see http://www.mouselabweb.org.

2 The definition of the scale can be found in the Sect. 3.

3 Both the primary and the secondary items are shown in Appendix "The items in the SVO task".
} 


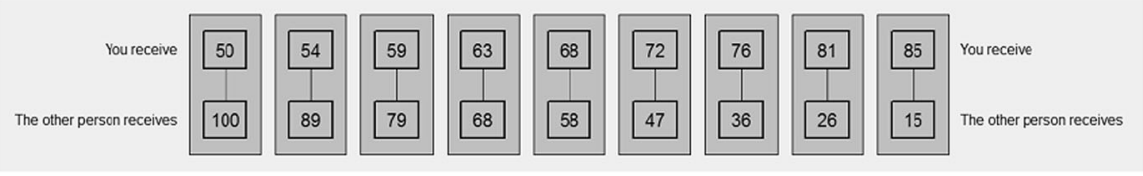

Fig. 1 The screenshot of one item in the SVO task (translated from German)

of the screen to get into the decision situation. Thus, the mouse was always positioned horizontally in the middle and vertically in the lower part of the screen immediately preceding each decision.

The experiment was computerized using z-Tree (Fischbacher 2007). A total of 132 students recruited via ORSEE (Greiner 2015) took part in the experiment between October and November 2014. The experiment took place in the Lakelab at the University of Konstanz. Upon entering the laboratory, subjects were randomly assigned into pairs. We randomized the order of the 15 items for each pair. At the end of the session, one of the 15 items was randomly selected to be paid out in each pair according to one person's choice, who was randomly determined as well. The SVO task was part of another experiment and conducted at the beginning of the session (see Instructions in the "Appendix"). The whole experiment lasted for about one and a half hours and the subjects earned 13.80 Euros on average.

\section{Results}

\subsection{Social value orientation}

All choice items in the SVO measure correspond to nine equidistant points on a line in the self/other allocation plane. The six primary items correspond to the six lines that connect four points on a circle with center $(50,50)$ and radius 50 . The points correspond to four SVO types: altruistic, prosocial, individualistic, and competitive types. The average of the chosen allocations in the primary items determines the SVO angle as follows:

$$
\text { SVO angle }=\arctan \left(\frac{\bar{P}_{O}-50}{\bar{P}_{S}-50}\right)
$$

where $\bar{P}_{S}$ is the average of the own payoff, and $\bar{P}_{O}$ is the average of the other's payoff in the chosen allocations. The SVO angle is a (practically) continuous measure of social value orientation and a higher angle indicates greater concern for the welfare of others. SVO angles of $-16.26^{\circ}, 0^{\circ}$ and $61.39^{\circ}$ indicate perfect competitiveness, perfect selfishness, and perfect altruism, respectively. Given the computed SVO angles, subjects can also be categorized into four types: altruists $\left(\mathrm{SVO}>57.15^{\circ}\right)$, prosocials $\left(22.45^{\circ}<\mathrm{SVO}<57.15^{\circ}\right)$, individualists $\left(-12.04^{\circ}<\right.$ $\mathrm{SVO}<22.45^{\circ}$ ), and competitors $\left(\mathrm{SVO}<-12.04^{\circ}\right) .^{4}$

\footnotetext{
${ }^{4}$ For further details, see Murphy et al. (2011).
} 


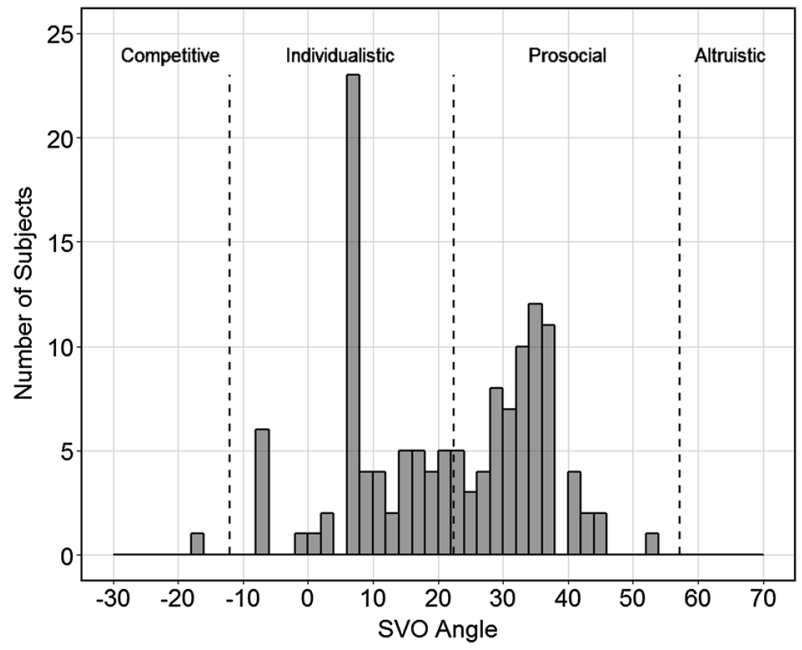

Fig. 2 The distribution of the SVO angles

Subjects' SVO angles are reported in Fig. 2. In line with the previous findings (Ackermann et al. 2016; Murphy et al. 2011), subjects vary considerably in their SVOs. In our study, the average SVO angle equals $21.69^{\circ}\left(\mathrm{sd} .=14.15^{\circ}\right)$ with the lowest observation being an angle of $-16.26^{\circ}$ and the highest observation being an angle of $52.91^{\circ}$. Among the 132 subjects, 68 (51.52\%) subjects are prosocial, 63 $(47.73 \%)$ subjects are individualistic, and $1(0.76 \%)$ subject is competitive.

\subsection{Response time and preferences}

Next we study the correlation between SVOs and response times. There are two reasons why we expect shorter response times for individualistic subjects in the SVO task. First, individualistic subjects have smaller information requirements because they do not care about the payoff of the other player. This has also been shown in eye tracking studies (Fiedler et al. 2013). Second, for the individualistic subjects the decision does not involve a conflict (or involves a weak conflict) between different motives while for prosocial subjects most of the decisions are associated with a stronger conflict between the own payoff and the payoff for the other player. It has been shown that the number of cognitive conflicts between motives and the degree of conflict lead to longer response times (Chen and Fischbacher 2015; Evans et al. 2015). Therefore, we expect that on average, the subjects with higher SVOs are slower in making decision in the SVO task. ${ }^{5}$

\footnotetext{
${ }^{5}$ If there are many subjects in each SVO type, the response time should increase from pure individualistic subjects to pure altruistic subjects, as well as from pure individualistic subjects to pure competitive subjects. That is, there should exist a U-relationship between response times and SVO angles. In the SVO slider measure, a perfectly consistent individualistic subject yields an angle between $-7.82^{\circ}$ and $7.82^{\circ}$. In our experiment, there are no altruistic subjects and only 1 subject whose SVO angle is less than $-7.82^{\circ}$. Therefore, we expect that, on average, the response time increases with the SVO angle in our study.
} 

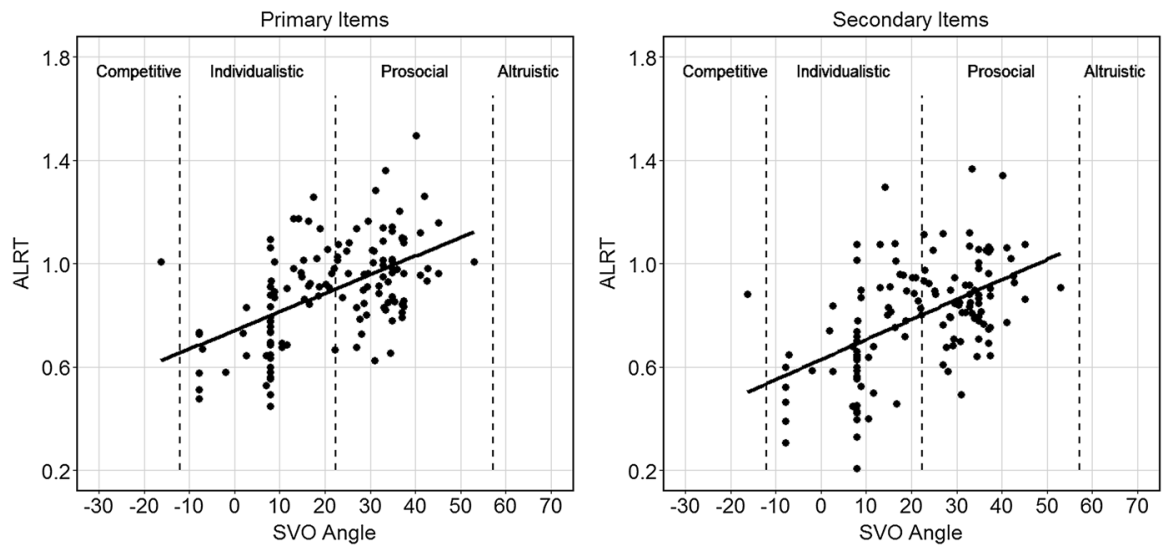

Fig. 3 The relationship between the average logarithm of response times and the SVO angles

The distributions of response times and common logarithm of response times are shown in Appendix "Distributions of response times and the common logarithm of response times". The figures show that the distribution of response time is right skewed, but the common logarithmic transformation makes it symmetric and removes outliers. ${ }^{6}$ The average of the logarithm of response times (ALRT) in the primary items is 0.898 with a standard deviation of 0.293 , and the ALRT in the secondary items is 0.797 with a standard deviation of 0.310 . The left panel of Fig. 3 shows the relationship between the ALRT in the primary items and the SVO angle for each subject. The response times are positively correlated with the SVO angles (Spearman two-sided test, $\rho=0.506, p<10^{-9}$ ). ${ }^{7}$ That is, the subjects with higher SVOs are slower in making decisions in the SVO task.

Result 1 The subjects who are more prosocial are slower in making decisions in the SVO task.

This result is based on the data of the primary items, which we use to determine the subjects' SVOs. The secondary items are used to assess the degree of inequality aversion vs. the degree of joint gain maximization among prosocial subjects. Thus, the data of the secondary items allows us to check the robustness of our first result. The right panel of Fig. 3 indicates that the response times in the secondary items are also positively correlated with the SVO angles (Spearman two-sided test, $\rho=0.514$, $p<10^{-9}$ ), which confirms our first finding. Moreover, regressions (1) and (2) in Table 1 show that the coefficients of SVO angle are significantly positive in both primary and secondary items for individualistic subjects, also if we control for the decision number and the distance that the cursor has to move from the button in the bottom-center to the chosen option. The coefficients of SVO angle are also positive for prosocial subjects (significant in the primary items, but not significant in the secondary items, $p=0.109$ ). The sign of the distance that the cursor has to move is negative,

\footnotetext{
${ }^{6}$ After the logarithm transformation, all observations lie within $(-2.792,3.403)$ standard deviations.

7 To test the robustness of our findings, we also conducted the analysis using the untransformed response times, which essentially led to the same results.
} 
Table 1 OLS regressions of response times

\begin{tabular}{lllll}
\hline & $\begin{array}{l}(1) \\
\text { Primary } \\
\text { items }\end{array}$ & $\begin{array}{l}\text { Secondary } \\
\text { items }\end{array}$ & $\begin{array}{l}(3) \\
\text { Primary items without } \\
\text { extreme decisions }\end{array}$ & $\begin{array}{l}\text { Secondary items without } \\
\text { extreme decisions }\end{array}$ \\
\hline SVO angle & $0.012 *$ & 0.012 & 0.012 & 0.008 \\
& $(0.007)$ & $(0.007)$ & $(0.012)$ & $(0.009)$ \\
Individualist & -0.243 & -0.261 & -0.358 & -0.099 \\
& $(0.241)$ & $(0.256)$ & $(0.396)$ & $(0.337)$ \\
SVO & $0.021^{* *}$ & $0.020^{* *}$ & $0.031 *$ & 0.016 \\
angle $\times$ individualist & $(0.009)$ & $(0.009)$ & $(0.018)$ & $(0.013)$ \\
Decision number & $-0.023 * * *$ & $-0.037 * * *$ & $-0.024 * *$ & $-0.034 * * *$ \\
Distance from the & $(0.005)$ & $(0.005)$ & $(0.012)$ & $(0.007)$ \\
bottom-center & $-0.014 * * *$ & $-0.012 * * *$ & -0.006 & $0.020^{* *}$ \\
Constant & $(0.004)$ & $(0.004)$ & $(0.014)$ & $(0.009)$ \\
& $4.239 * * *$ & $3.886 * * *$ & 3.074 & -1.093 \\
$R^{2}$ & $(0.611)$ & $(0.659)$ & $(2.343)$ & $(1.461)$ \\
Adj. $R^{2}$ & 0.192 & 0.220 & 0.142 & 0.081 \\
Num. obs. & 0.187 & 0.217 & 0.123 & 0.073 \\
\hline
\end{tabular}

The dependent variable is $\ln (R T)$. Individualist is a dummy variable for SVO type (1 for individualistic type and 0 for prosocial type). Decision Number is the number of the decision situation which measures the subjects' experiences. Distance from the Bottom-Center is the linear distance that the cursor has to move from the continue button in the bottom-center to the center of the chosen option. The distance is measured using the same unit as the click position analysis. The robust standard errors are clustered on each subject and reported in parentheses

$* * * p<0.01, * * p<0.05, * p<0.1$

which is counterintuitive at first glance. The reason is that the extreme decisions are easier to make. This is consistent with a view that at least some subjects were willing to take an even more extreme decision and, therefore, the decision is particularly easy. In regressions (3) and (4) of Table 1, we exclude the decisions in which the leftmost or the rightmost options were chosen. The results show that the distance has no significant effect on response time in the primary items and has significantly positive effect on response time in the secondary items. The horizontal extent of the decision area is larger than the vertical extent and only this direction directly affects the decision. Nevertheless, the vertical click position could affect decision time. Because the own payoff was also on top and further away from the initial mouse position, we would expect that selfish decisions would be slower. We analyzed this question in a regression analysis and found that it is not the case. If anything, decisions in the upper part are quicker but this effect is far from significant.

\subsection{Click position and preferences}

Now, we turn to the correlation between the click positions and people's preferences. In the experiment, the subjects make decisions by clicking on the 

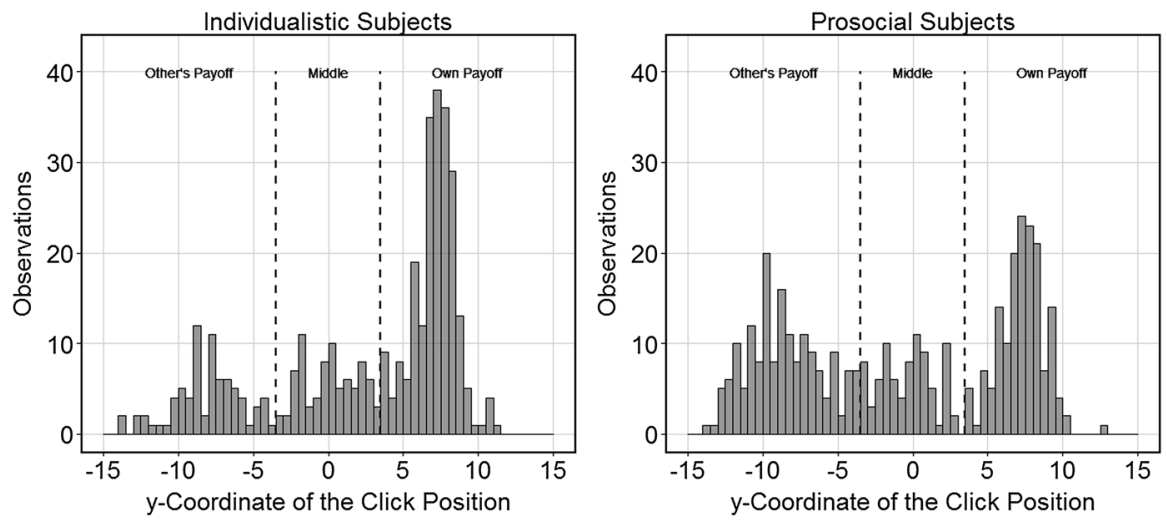

Fig. 4 Distributions of the $y$ coordinates of the click positions of individual decisions in the primary items, separated for individualistic and prosocial subjects. The $y$ coordinate is normalized to the center of the clickable area

options they prefer most. The option contains the own as well as the other player's payoff. The click position reveals the focus of people's attention in the final stage of the decision. Our main interest is in whether the subjects click on their own or the others' payoffs. Thus, we focus on the $y$ coordinates of the click positions. We expect that individualistic subjects put most attention on their own payoffs since they care more about their own payoffs, while prosocial subjects distribute their attention among both payoffs since they care about both their own payoffs and the others' payoffs (Fiedler et al. 2013; Jiang et al. 2016). ${ }^{8}$

Figure 4 shows histograms of the $y$ coordinates of the click positions of individual decisions for the primary items. Because the $y$ coordinate is normalized to the center of Fig. 1, the $y$ coordinate of the own payoffs is positive and the $y$ coordinate of the other's payoffs is negative. Figure 4 shows that most of the click positions of individualistic subjects are located in the areas of their own payoffs, while a higher proportion of the click positions of prosocial subjects are located in the areas of the others' payoffs. For a statistical analysis, we count how many times each subject clicks on her own payoff, the other's payoff and the middle areas between her own payoff and the other's payoff. Figure 5 shows distributions of these frequency combinations for individualistic and prosocial subjects, respectively. For example, 27 of the individualistic subjects always click in the regions of the own payoff, 4 always click in the regions of the other player's payoff and 2 always click in the middle areas. Of the 63 individualistic subjects, 41 subjects click more often on the own payoff and only 18 click more often on the other's payoff. These frequencies are significantly different (two-sided binomial test, $p=0.004$ ). This is different for prosocial subjects. Of the 68 prosocial subjects, 27 subjects click more often on the own payoff and 31 click more often on the other's payoff. So prosocial subjects click even more frequently on the payoff of the other player but the frequencies are not significantly different (two-sided binomial test,

\footnotetext{
8 There is only 1 competitive subject in our experiment. We omit this subject in the click position analysis.
} 

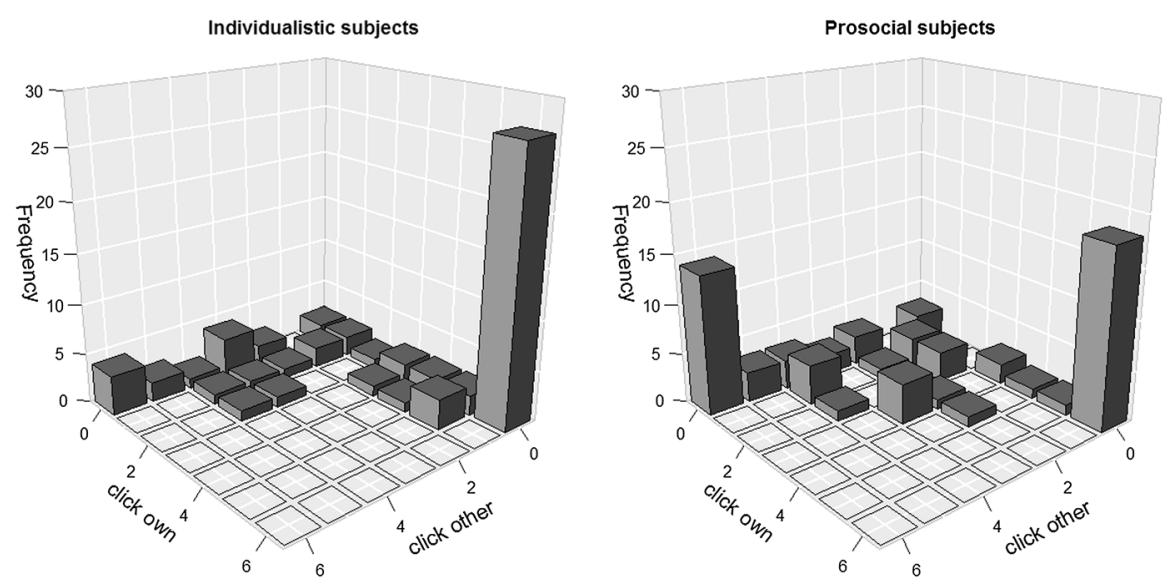

Fig. 5 The frequency combinations of clicking own vs. the other's payoff in the primary items, separated for individualistic and prosocial subjects

$p=0.694)$. We can also directly compare the distribution of the individualistic subjects with the distribution of the prosocial subjects. Individualistic subjects click significantly more on their own payoffs than prosocial subjects, they click significantly less often on the others' payoffs than prosocial subjects, and the number of times that subjects click on the middle areas are not significantly different between the two groups (two-sided bootstrap Kolmogorov-Smirnov tests, $p=0.014, p=0.007$ and $p=0.994$, respectively). That is, individualistic subjects click more often on their own payoffs than on the others' payoffs; and they click more often on their own payoffs than prosocial subjects.

Result 2 Individualistic subjects click more often on their own payoffs than on the others' payoffs; and they click more often on their own payoffs than prosocial subjects.

Similar to the response time analysis, we can also use the data of the secondary items to check the robustness of our result regarding the click positions. Figure 6 displays histograms of the $y$ coordinates of the click positions in the secondary items. As in the primary items, most of the click positions of individualistic subjects are located in the regions of their own payoffs, while a higher proportion of the click positions of prosocial subjects are located in the regions of the others' payoffs. Figure 7 shows distributions of the frequency combinations of the click positions in the secondary items, separately for individualistic and prosocial subjects. Also for the secondary items, the majority of individualistic subjects click more frequently on the own payoff, 39 subjects in comparison to 23 who click more frequently on the other player's payoff. These frequencies are marginally significantly different (two-sided binomial test, $p=0.056$ ). This is also different for prosocial subjects. Of the 68 prosocial subjects, 24 subjects clicked more often on the own payoff and 38 clicked more often on the other's payoff. Prosocial subjects click more frequently on the payoff of the other player but the frequencies are only marginally statistically different (two-sided binomial test, $p=0.098$ ). Nevertheless, the two distributions are significantly different. Individualistic subjects click significantly more often on 

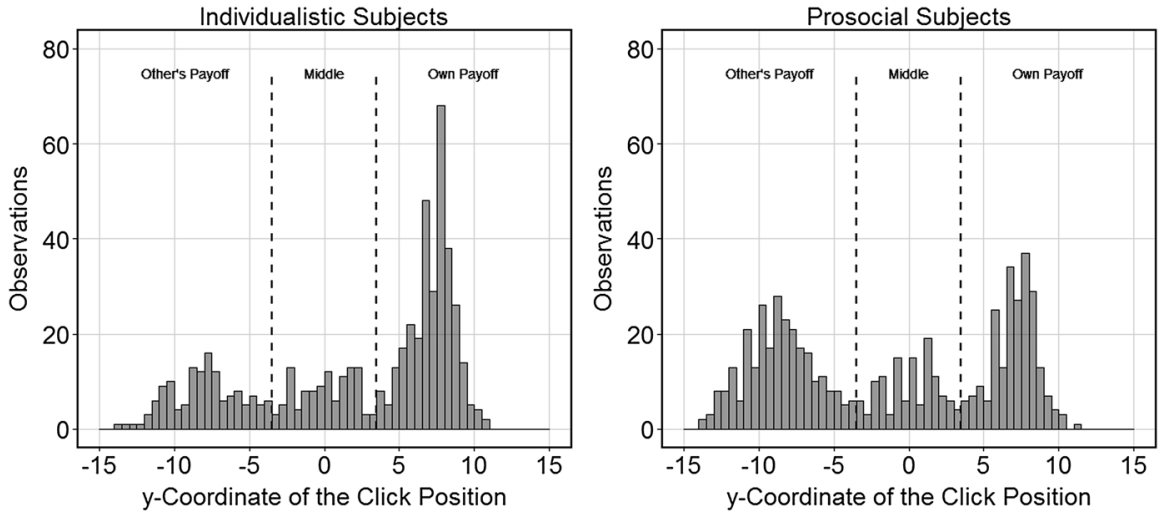

Fig. 6 Distributions of the $y$ coordinates of the click positions of individual decisions in the secondary items, separated for individualistic and prosocial subjects. The $y$ coordinate is normalized to the center of the clickable area
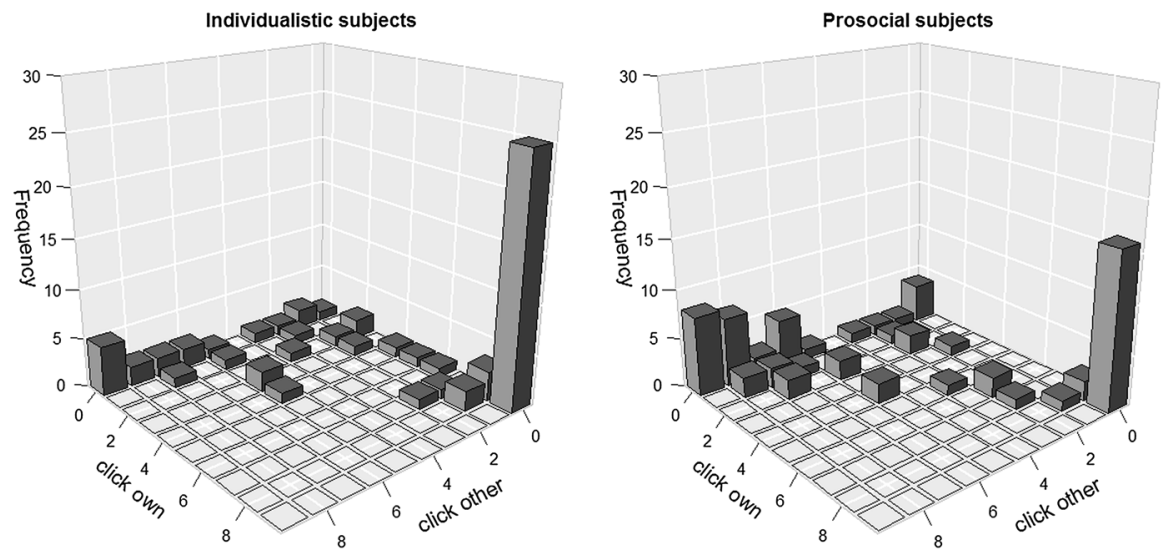

Fig. 7 The frequency combinations of clicking own vs. the other's payoff in the secondary items, separated for individualistic and prosocial subjects

their own payoffs than prosocial subjects, they click significantly less often on the others' payoffs than prosocial subjects, and the number of times that subjects click on the middle area are not significantly different between the two groups (two-sided bootstrap Kolmogorov-Smirnov tests, $p=0.022, p=0.003$ and $p=0.777$, respectively). That is, individualistic subjects click more often on their own payoffs than on the others' payoffs; and they click more often on their own payoffs than prosocial subjects. Thus, the data of the secondary items confirm the robustness of our result regarding the click positions.

We conclude this subsection with an analysis of whether the correlation between click position pattern and SVO type is driven by the type of the subject or by the individual decision. In all items that are used to calculate the SVO angle, the other player's payoff varies linearly. This makes it possible to define an altruism measure 
Table 2 Logit regressions of altruism of decision and SVO angle on click position in the primary items

\begin{tabular}{llll}
\hline & $(1)$ & $(2)$ & $(3)$ \\
\hline Constant & $0.625 * * *$ & $1.080^{* * *}$ & $1.127 * * *$ \\
& $(0.171)$ & $(0.296)$ & $(0.299)$ \\
Degree of altruism of decision & $-0.750^{* * *}$ & & $-0.349 * *$ \\
& $(0.188)$ & & $(0.153)$ \\
SVO angle & & $-0.034 * * *$ & $-0.030^{* * *}$ \\
& & $(0.011)$ & $(0.011)$ \\
AIC & 881.328 & 863.523 & 862.833 \\
BIC & 890.297 & 872.492 & 876.287 \\
Log likelihood & -438.664 & -429.761 & -428.417 \\
$R^{2}$ & 0.018 & 0.037 & 0.041 \\
Num. obs. & 655 & 655 & 655 \\
\hline
\end{tabular}

The dependent variable is a dummy variable of clicking on the upper part (1: own payoff) or the lower part (0: other's payoff). Item 2 is excluded (selfishness and altruism aligned). The robust standard error are clustered on each subject and reported in parentheses

$* * * p<0.01, * * p<0.05, * p<0.1$

for every decision. It equals one for the most altruistic choice, zero for the most selfish choice and is linearly interpolated for the values in between. In Table 2, we show how these measures affect whether the own payoff is clicked or not. The regressions show that both the type of the subject measured with the SVO angle as well as the individual decision are important but the type of the subject is more important. It has a higher $R^{2}$ as a single explanatory variable and it is more robust if we include the other variable.

\subsection{Comparison of response time and click position}

In this part, we investigate how much of the variation in preferences can be explained by response time and click position, and whether one is redundant if the other is available. First, we calculate the classification rate when assigning a subject to a prosocial or an individualistic type using only the response time measure (ALRT) or the click position measure (number of clicks on the other's payoff). As there are 63 individualistic and 68 prosocial subjects according to the classification standard of the SVO task, we use the $0.48[63 /(63+68)]$ quantile of the response time measure or the click position measure as the classification boundary. The classification results in Table 3 show that the click position works almost equally as well as the response time. For instance, in the primary items, 40 of 63 subjects who are below or equal to the response time boundary are individualistic, and 45 of 68 subjects who are above or equal to the response time boundary are prosocial. With respect to the click position, 38 of 63 subjects who are below or equal to the click position boundary are individualistic, and 43 of 68 subjects who are above or equal to the click position boundary are prosocial.

Next we compare the explanatory power of response time and click position on subjects' preferences. Specifically, we use response time (ALRT) and click position 
Table 3 Classification based on response time or click position

\begin{tabular}{|c|c|c|c|c|}
\hline & \multicolumn{2}{|l|}{ Primary items } & \multicolumn{2}{|c|}{ Secondary items } \\
\hline & Individualistic & Prosocial & Individualistic & Prosocial \\
\hline \multicolumn{5}{|l|}{ Response time } \\
\hline Individualistic (63) & 40 & 23 & 42 & 21 \\
\hline Prosocial (68) & 23 & 45 & 21 & 47 \\
\hline \multicolumn{5}{|l|}{ Click position } \\
\hline Individualistic (63) & 38 & 25 & 41 & 25 \\
\hline Prosocial (68) & 25 & 43 & 29 & 46 \\
\hline
\end{tabular}

Table 4 Logit regressions of response time and click position information on SVO type

\begin{tabular}{|c|c|c|c|c|c|c|}
\hline & \multicolumn{3}{|c|}{ Primary items } & \multicolumn{3}{|c|}{ Secondary items } \\
\hline & (1) & (2) & (3) & (4) & $(5)$ & (6) \\
\hline ALRT & $\begin{array}{l}5.111^{* * *} \\
(1.173)\end{array}$ & & $\begin{array}{l}5.180 * * * \\
(1.222)\end{array}$ & $\begin{array}{l}4.983 * * * \\
(1.127)\end{array}$ & & $\begin{array}{l}4.942 * * * \\
(1.155)\end{array}$ \\
\hline $\begin{array}{l}\text { Number of clicks on the } \\
\text { other's payoff }\end{array}$ & & $\begin{array}{l}0.265^{* * *} \\
(0.086)\end{array}$ & $\begin{array}{l}0.273 * * * \\
(0.096)\end{array}$ & & $\begin{array}{l}0.157 * * * \\
(0.054)\end{array}$ & $\begin{array}{l}0.152 * * \\
(0.060)\end{array}$ \\
\hline Constant & $\begin{array}{l}-4.496 * * * \\
(1.067)\end{array}$ & $\begin{array}{l}-0.398^{*} \\
(0.233)\end{array}$ & $\begin{array}{l}-5.045^{* * *} \\
(1.144)\end{array}$ & $\begin{array}{l}-3.889 * * * \\
(0.920)\end{array}$ & $\begin{array}{l}-0.401 * \\
(0.240)\end{array}$ & $\begin{array}{l}-4.310 * * * \\
(0.970)\end{array}$ \\
\hline AIC & 160.890 & 175.059 & 153.992 & 159.248 & 176.461 & 154.480 \\
\hline $\mathrm{BIC}$ & 166.641 & 180.809 & 162.618 & 164.998 & 182.211 & 163.106 \\
\hline Log likelihood & -78.445 & -85.529 & -73.996 & -77.624 & -86.230 & -74.240 \\
\hline$R^{2}$ & 0.135 & 0.057 & 0.184 & 0.144 & 0.049 & 0.182 \\
\hline Num. obs. & 131 & 131 & 131 & 131 & 131 & 131 \\
\hline
\end{tabular}

This analysis does not include the competitive subject. The dependent variable is a dummy variable for SVO type (1 for prosocial and 0 for individualistic type). ALRT is the average logarithm of response times for the subject. Number of clicks on the other's payoff is how many times the subject clicked on the other's payoff

$* * * p<0.01, * * p<0.05, * p<0.1$

information (number of clicks on the other's payoff) to predict subjects' SVO type. ${ }^{9}$ Table 4 shows the regression results. The dependent variable is a dummy variable for SVO type (1 for prosocial and 0 for individualistic type). In both primary and secondary items, the regressions which includes both response time and click position have higher explanatory power than the regressions in which only response time or click position is included. We use regressions (3) and (6) to predict subjects' SVO type, and the results are shown in Table 5. The classification in Table 5 is clearly better than the classification in Table 3. To compare the classifications in Table 5 and Table 3 quantitatively, we calculate the $\varphi$ coefficient. The $\varphi$ coefficient

\footnotetext{
9 We can get similar results if we use response time and click position to predict the SVO angle, results are shown in Table 6 in "Appendix".
} 
Table 5 Classification based on both response time and click position

\begin{tabular}{llllll}
\hline & \multicolumn{2}{l}{ Primary items } & & \multicolumn{2}{l}{ Secondary items } \\
\cline { 2 - 3 } \cline { 5 - 6 } & Individualistic & Prosocial & & Individualistic & Prosocial \\
\hline Individualistic (63) & 44 & 19 & & 43 & 20 \\
Prosocial (68) & 19 & 49 & & 20 & 48 \\
\hline
\end{tabular}

for the primary items in Table 5 equals 0.419 , which is higher than the $\varphi$ coefficient if we only use response time (0.297) or click position (0.236) in Table 3 . The $\varphi$ coefficient for the secondary items in Table 5 equals 0.388 , which is also higher than the $\varphi$ coefficient if we only use response time $(0.358)$ or click position $(0.234)$ in Table 3. This is not surprising because the two measures are only weakly and insignificantly correlated (Spearman correlation test, $\rho=0.109, p=0.213$ for primary items, and $\rho=0.110, p=0.213$ for the secondary items).

Result 3 Response time information and click position information are complementary in explaining subjects' social value orientation.

\section{Conclusion}

This paper explores the usefulness of response time and click position in economic experiments. The results of the SVO experiment confirm earlier results that more prosocial people have longer response times. Our novel results concern click position. We find that the click position provides information about people's social preferences, i.e., it reveals information what features (own or other's payoff) people care about. Individualistic subjects click more often on their own payoffs than on the others' payoffs and they are more likely to click on their own payoffs than prosocial subjects. Response time information and click position information are complementary in explaining subjects' preferences. Furthermore, data of the secondary items of the SVO task confirms the robustness of our results.

Our results are relevant since data about response times and click positions can be collected at almost no cost in lab or online experiments and it is wasteful to not make use of them. Our setting investigates the relevance of different attributes for social preferences. However, the general idea can be applied to any type of choice between multi-attribute options such as consumer decisions, where options differ in price and brand attributes. Also, in these kinds of decisions response time data and click positions provide additional information about people's preferences and can be used to improve the predictive power. Process data can enhance the analysis of the decision by providing additional evidence that can be economically relevant. For example, response times can provide information about the intuitiveness or difficulty of a decision, and the click position can deliver evidence of potential motives for a decision in addition to the decision itself.

Acknowledgments We would like to thank Dominik Bauer, David Dohmen, Fabian Dvorak, David Grammling, Thomas Hattenbach, Jan Hausfeld, Konstantin Hesler, Katrin Schmelz, and Irenaeus Wolf 
for helpful comments and discussions. The authors gratefully acknowledge financial support from the German Research Foundation (DFG) through research unit FOR 1882 "Psychoeconomics".

\section{Appendix}

\section{The items in the SVO task}

\section{Six primary items}

See Fig. 8.

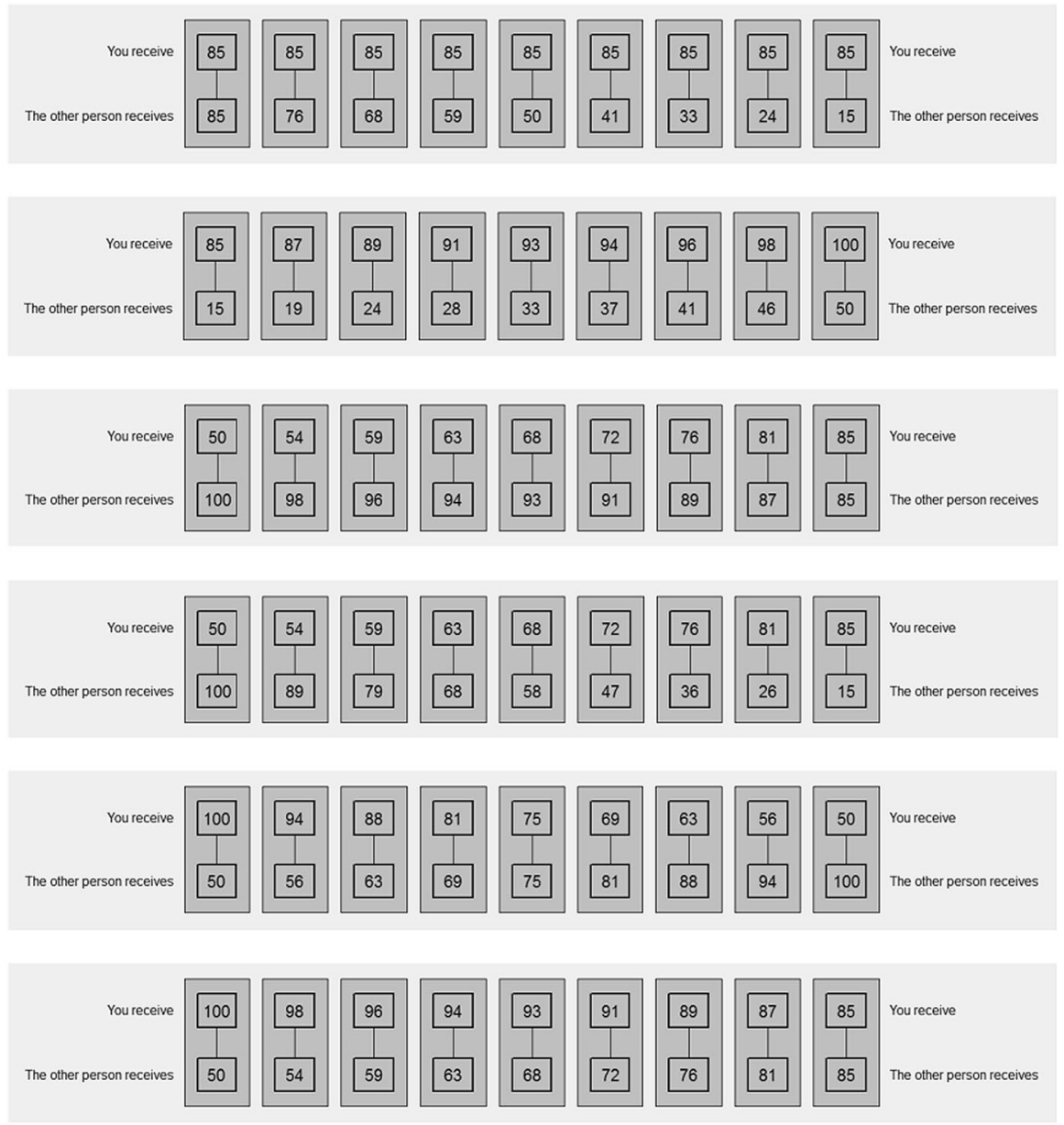

Fig. 8 The primary items 


\section{Nine secondary items}

See Fig. 9.

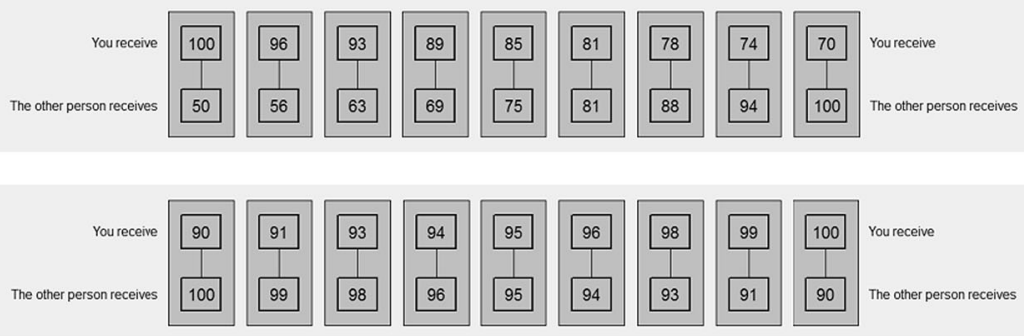

\begin{tabular}{|c|c|c|c|c|c|c|c|c|c|c|}
\hline You receive & 100 & 94 & 88 & 81 & 75 & 69 & 63 & 56 & 50 & You receive \\
\hline The other person receives & 70 & 74 & 78 & 81 & 85 & 89 & 93 & 96 & 100 & The other person receives \\
\hline
\end{tabular}

\begin{tabular}{|c|c|c|c|c|c|c|c|c|c|c|}
\hline You receive & 100 & 99 & 98 & 96 & 95 & 94 & 93 & 91 & 90 & You receive \\
\hline he other person receives & 70 & 74 & 78 & 81 & 85 & 89 & 93 & 96 & 100 & The other person receives \\
\hline
\end{tabular}

\begin{tabular}{|c|c|c|c|c|c|c|c|c|c|c|}
\hline You receive & 70 & 74 & 78 & 81 & 85 & 89 & 93 & 96 & 100 & You receive \\
\hline The other person receives & 100 & 96 & 93 & 89 & 85 & 81 & 78 & 74 & 70 & The other person receives \\
\hline
\end{tabular}

\begin{tabular}{|c|c|c|c|c|c|c|c|c|c|c|}
\hline You receive & 50 & 56 & 63 & 69 & 75 & 81 & 88 & 94 & 100 & You receive \\
\hline The other person receives & 100 & 99 & 98 & 96 & 95 & 94 & 93 & 91 & 90 & The other person receives \\
\hline
\end{tabular}
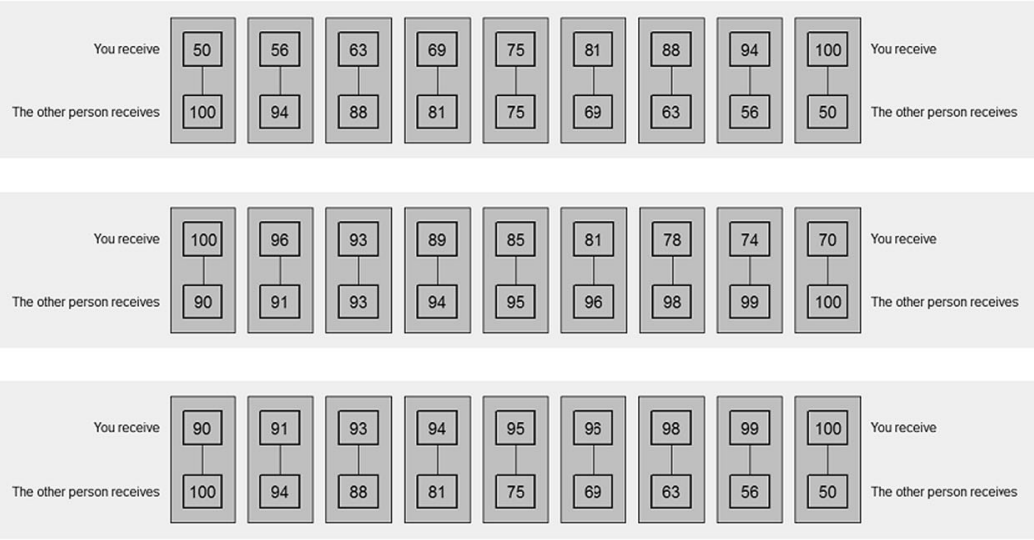

Fig. 9 The secondary items 


\section{Distributions of response times and the common logarithm of response times}

\section{Distributions of response times}

See Fig. 10.
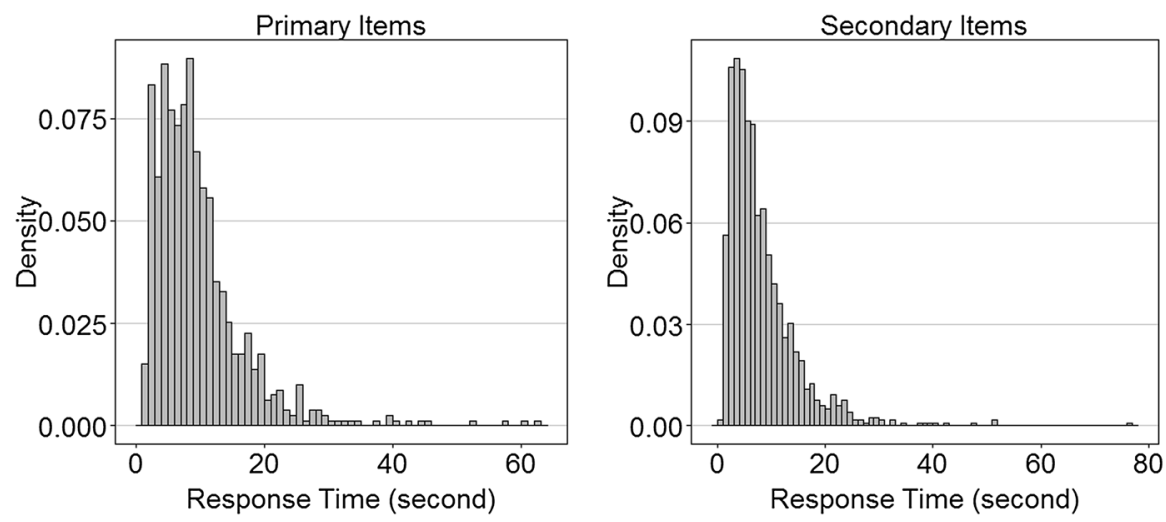

Fig. 10 Distributions of response times in the primary and secondary items

Distributions of the common logarithm of response times

See Fig. 11.
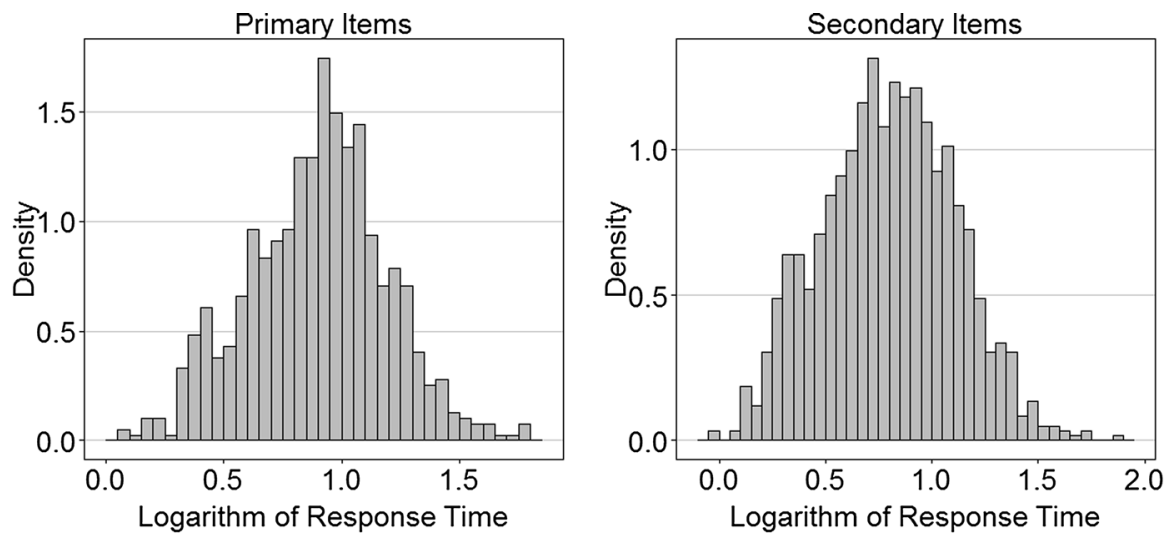

Fig. 11 Distributions of the common logarithm of response times in the primary and secondary items 


\section{Compare the explanatory power of response time and click position on SVO angle}

See Table 6.

Table 6 OLS regressions of response time and click position information on SVO angle

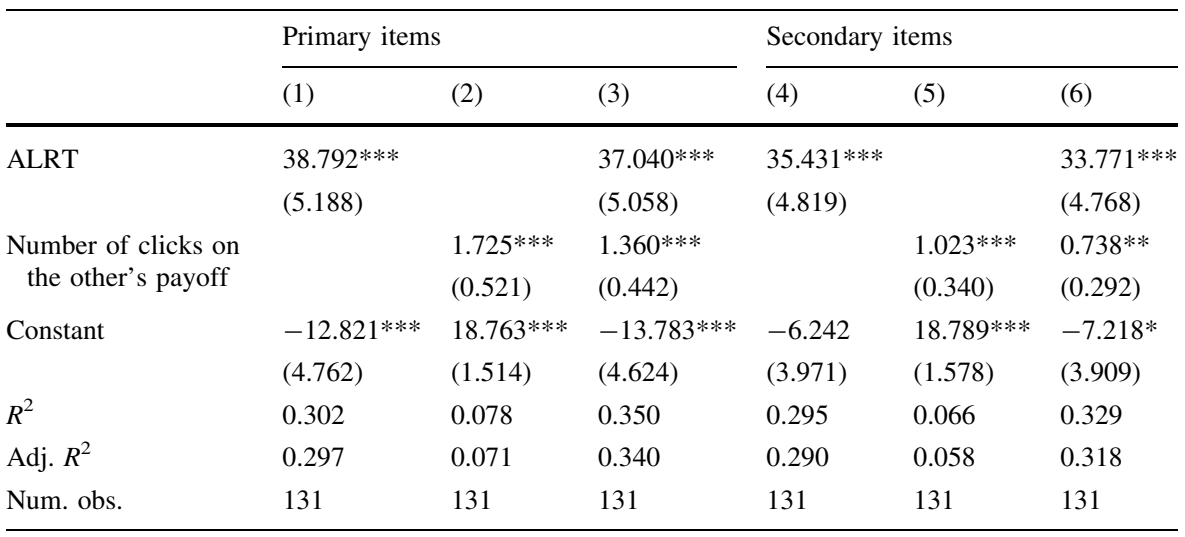

This analysis does not include the competitive subject. The dependent variable is the SVO angle ALRT is the average logarithm of response time for the subject. Number of clicks on the other's payoff is how many times the subject clicked on the other's payoff

$* * * p<0.01, * * p<0.05, * p<0.1$

\section{References}

Achtziger, A., \& Alós-Ferrer, C. (2014). Fast or rational? A response-times study of Bayesian updating. Management Science, 60(4), 923-938.

Ackermann, K. A., Fleiß, J., \& Murphy, R. O. (2016). Reciprocity as an individual difference. Journal of Conflict Resolution, 60(2), 340-367.

Brocas, I., Carrillo, J. D., Wang, S. W., \& Camerer, C. F. (2014). Imperfect choice or imperfect attention? Understanding strategic thinking in private information games. Review of Economic Studies, 81(3), 944-970.

Chen, F., \& Fischbacher, U. (2015). Cognitive processes of distributional preferences: A response time study. TWI Working Paper.

Evans, A. M., Dillon, K. D., \& Rand, D. G. (2015). Fast but not intuitive, slow but not reflective: Decision conflict drives reaction times in social dilemmas. Journal of Experimental Psychology: General, 144(5), 951-966.

Fiedler, S., Glöckner, A., Nicklisch, A., \& Dickert, S. (2013). Social value orientation and information search in social dilemmas: An eye-tracking analysis. Organizational Behavior and Human Decision Processes, 120(2), 272-284.

Fischbacher, U. (2007). z-Tree: Zurich toolbox for ready-made economic experiments. Experimental Economics, 10(2), 171-178.

Greiner, B. (2015). Subject pool recruitment procedures: Organizing experiments with ORSEE. Journal of the Economic Science Association, 1(1), 114-125.

Guo, F., Li, L., \& Faloutsos, C. (2009). Tailoring click models to user goals. Paper read at Proceedings of the 2009 workshop on Web Search Click Data.

Hutcherson, C. A., Bushong, B., \& Rangel, A. (2015). A neurocomputational model of altruistic choice and its implications. Neuron, 87(2), 451-462. 
Jiang, T., Potters, J., \& Funaki, Y. (2016). Eye tracking social preferences. Journal of Behavioral Decision Making, 29, 157-168.

Kieslich, P. J., \& Hilbig, B. E. (2014). Cognitive conflict in social dilemmas: An analysis of response dynamics. Judgment \& Decision Making, 9(6), 510-522.

Koop, G. J., \& Johnson, J. G. (2011). Response dynamics: A new window on the decision process. Judgment \& Decision Making, 6(8), 750-758.

Krajbich, I., Armel, C., \& Rangel, A. (2010). Visual fixations and the computation and comparison of value in simple choice. Nature Neuroscience, 13(10), 1292-1298.

Krajbich, I., Bartling, B., Hare, T., \& Fehr, E. (2015). Rethinking fast and slow based on a critique of reaction-time reverse inference. Nature Communications, 6, 7455.

Krajbich, I., Oud, B., \& Fehr, E. (2014). Benefits of neuroeconomic modeling: new policy interventions and predictors of preference. American Economic Review, 104(5), 501-506.

Liebrand, W. B. G., \& McClintock, C. G. (1988). The ring measure of social values: A computerized procedure for assessing individual differences in information processing and social value orientation. European Journal of Personality, 2(3), 217-230.

Liu, J., Dolan, P., \& Pedersen, E. R. (2010). Personalized news recommendation based on click behavior. Paper read at Proceedings of the 15th international conference on intelligent user interfaces.

Murphy, R. O., Ackermann, K. A., \& Handgraaf, M. J. J. (2011). Measuring social value orientation. Judgment \& Decision Making, 6(8), 771-781.

Payne, J. W., Bettman, J. R., \& Johnson, E. J. (1993). The Adaptive Decision Maker. New York: Cambridge University Press.

Piovesan, M., \& Wengström, E. (2009). Fast or fair? A study of response times. Economics Letters, 105(2), 193-196.

Rand, D. G., Greene, J. D., \& Nowak, M. A. (2012). Spontaneous giving and calculated greed. Nature, 489(7416), 427-430.

Reutskaja, E., Nagel, R., Camerer, C. F., \& Rangel, A. (2011). Search dynamics in consumer choice under time pressure: An eye-tracking study. American Economic Review, 101(2), 900-926.

Rubinstein, A. (2007). Instinctive and cognitive reasoning: A study of response times. The Economic Journal, 117(523), 1243-1259.

Schulz, J. F., Fischbacher, U., Thöni, C., \& Utikal, V. (2014). Affect and fairness: Dictator games under cognitive load. Journal of Economic Psychology, 41, 77-87.

Smith, A., Bernheim, B. D., Camerer, C. F., \& Rangel, A. (2014). Neural activity reveals preferences without choices. American Economic Journal: Microeconomics, 6(2), 1-36.

Spiliopoulos, L., \& Ortmann, A. (2015). The BCD of response time analysis in experimental economics. Working Paper. SSRN 2401325.

Spivey, M. J., Grosjean, M., \& Knoblich, G. (2005). Continuous attraction toward phonological competitors. Proceedings of the National Academy of Sciences of the United States of America, 102(29), 10393-10398.

Wang, Joseph Tao-Yi, Spezio, Michael, \& Camerer, Colin F. (2010). Pinocchio's pupil: Using eyetracking and pupil dilation to understand truth telling and deception in sender-receiver games. American Economic Review, 100(3), 984-1007. 